\title{
Conformal and Disformal Structure of 3D Circularly Symmetric Static Metric in $\mathbf{f}(\mathbf{R})$ Theory of Gravity
}

\author{
MUHAMMAD RAMZAN*, MURTAZA ALI*, AND FIAZ HUSSAIN*
}

RECEIVED ON 26.09.2018, ACCEPTED ON 19.02.2019

\begin{abstract}
Conformal vector fields are treated as generalization of homothetic vector fields while disformal vector fields are defined through disformal transformations which are generalization of conformal transformations, therefore it is important to study conformal and disformal vector fields. In this paper, conformal and disformal structure of 3D (Three Dimensional) circularly symmetric static metric is discussed in the framework of $f(R)$ theory of gravity. The purpose of this paper is twofold. Firstly, we have found some dust matter solutions of EFEs (Einstein Field Equations) by considering 3D circularly symmetric static metric in the $f(R)$ theory of gravity. Secondly, we have found CKVFs (Conformal Killing Vector Fields) and DKVFs (Disformal Killing Vector Fields) of the obtained solutions by means of some algebraic and direct integration techniques. A metric version of $f(R)$ theory of gravity is used to explore the solutions and dust matter as a source of energy momentum tensor. This study reveals that no proper DVFs exists. Here, DVFs for the solutions under consideration are either HVFs (Homothetic Vector Fields) or KVFs (Killing Vector Fields) in the $\mathbf{f}(\mathrm{R})$ theory of gravity. In this study, two cases have been discussed. In the first case, both CKVFs and DKVFs become HVFs with dimension three. In the second case, there exists two subcases. In the first subcase, DKVFs become HVFs with dimension seven. In the second subcase, CKVFs and DKVFs become KVFs having dimension four.
\end{abstract}

Key Words: Conformal Killing Vector Fields, Disformal Killing Vector Fields, Direct Integration Technique, $\mathbf{f}(\mathbf{R})$ Theory of Gravity.

\section{INTRODUCTION}

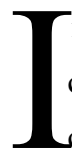
n physics, the relation between symmetries and conservation laws has fundamental importance. To classify the solutions of EFEs symmetries play an important role as with the help of symmetries one can explain geometry of spacetime [1]. Conformal symmetry which gives extra conservation laws than the other symmetry restrictions like Killing symmetry, homothetic symmetry etc. plays a key role in Einstein theory of GR (General Relativity). It is to be noted that Killing symmetry and homothetic motions are particular forms of conformal symmetry. Conformal symmetry for different spacetimes has been discussed by different researchers [2-3]. Hall and Capocci [4] present a classification and conformal symmetry in 3D metric. 3D metrics play a key

Authors E-Mail: (muhammad.ramzan@iub.edu.pk, murtazactn@gmail.com, mfiazbhutta@gmail.com

* Department of Mathematics, The Islamia University of Bahawalpur, Bahawalpur, Pakistan.

This is an open access article published by Mehran University Research Journal of Engineering and Technology, Jamshoro under the CC by 4.0 International License. 
role in physics especially to black hole thermodynamics. Taking attention to black hole configuration, BerredoPeixoto considered 3D gravity coupled to scalar field [5]. Banados et. al. [6] first discovered black hole solutions in 3D. After that a number of black hole solutions in 3D gravity have been found, a detail of which can be seen in [7-8] and the references therein. Bokhari et. al. [9] classified 3D circularly symmetric static metrics according to their isometries. Zhang et. al. [10] discussed black holes and gravitational waves in 3D $\mathrm{f}(\mathrm{R})$ gravity. Ali et. al. [11] investigated proper homothetic vector fields of $3 \mathrm{D}$ circularly symmetric static metric. Recently, Ramzan et. al. [12] discussed Ricci and Matter Collineations of Som-Roy Chaudhary Spacetime. Very recently, Larley [13] introduced the concept of DKVFs as a generalization of CKVFs. Actually, DKVFs are defined by means of disformal transformations which have many applications in TeVes theories for Mond [14], biometric theories of gravity [15], scalar tensor theories [16-19], disformal inflation [20], rainbow gravity [21] and many others. Keeping in mind the important applications of disformal transformations, we have used this idea in the $\mathrm{f}(\mathrm{R})$ theory of gravity which is introduced to justify current cosmic acceleration, problem of singularity, dark energy and dark matter issues etc. [22]. To tackle such type of issues, study of exact solutions in $\mathrm{f}(\mathrm{R})$ gravity has remained an important source of motivation for research. Therefore, working on 3D gravity theory plays a significant role in $3 \mathrm{D}$ physics. Our purpose in this paper is to find CKVFs and DKVFs by means of 3D geometry.

The rest of this paper is planned as follows: In section 2, we will give a brief derivation of dust matter solutions of EFEs by considering $3 \mathrm{D}$ circularly symmetric static metric in the $\mathrm{f}(\mathrm{R})$ theory of gravity as well as CKVFs and DKVFs of the resulting solutions. Section 3 is specified for results and discussion.

\section{FIELD EQUATIONS AND THEIR SOLUTIONS IN F(R) GRAVITY}

Modified field equations in $\mathrm{f}(\mathrm{R})$ gravity are [23].

$\mathrm{H}_{\mathrm{ab}}=\mathrm{kT}_{\mathrm{ab}}$

Where $H_{a b}=f(R) R_{a b}-\frac{1}{2} f(R) g_{a b}-\nabla_{a} \nabla_{b} F(R)+g_{a b} F(R), f(R)$ is the function of Ricci scalar $R, F(R)=\frac{d}{d R} f(R), k$ is the coupling constant, $T_{a b}$ is the standard energy momentum tensor and $\nabla \equiv \nabla^{\mathrm{a}} \nabla_{\mathrm{a}}$ in which $\nabla$ is the covariant derivative operator. To solve Equation (1), we consider 3D circularly symmetric static metric in the usual coordinates $(t, r, \theta)$ given by $\left(x^{0}, x^{1}, x^{2}\right)$ respectively [5].

$d s^{2}=e^{Y(r)} d t^{2}+e^{Z(r)} d r^{2}+r^{2} d \theta^{2}$

where $Y(r)$ and $Z(r)$ are non-zero functions of the radial coordinater. Non-zero components of Christofell symbols for the above metric Equation (2) are:

$\Gamma_{01}^{0}=\frac{1}{2} Y^{\prime}, \Gamma_{00}^{1}=\frac{1}{2} Y^{\prime} e^{Y-Z}, \Gamma_{11}^{1}=\frac{1}{2} Z^{\prime}, \Gamma_{22}^{1}=r e^{-Z}$, and $\Gamma_{12}^{2}=\frac{1}{r}$

Similarly, non-zero components of Ricci tensors for the above metric Equation (2) are:

$$
\left.\begin{array}{l}
R_{00}=\frac{1}{4 r}\left[2 r Y^{\prime \prime}+r Y^{\prime 2}-r Y^{\prime} Z^{\prime}+2 Y^{\prime}\right] e^{Y-Z} \\
R_{11}=\frac{1}{4 r}\left[2 r Y^{\prime \prime}+r Y^{\prime 2}-r Y^{\prime} Z^{\prime}+2 Z^{\prime}\right] \\
R_{22}=\frac{r}{2}\left[Y^{\prime}-Z^{\prime}\right] e^{-Z}
\end{array}\right\}
$$

where prime denotes the derivative with respect to the radial coordinate $r$. To explore solutions of Equation (1), we use dust matter as a source of energy momentum tensor i.e. $\mathrm{T}_{\mathrm{ab}}=\rho \mathrm{S}_{\mathrm{a}} \mathrm{S}_{\mathrm{b}}$ [23], where $\rho$ is the matter density and $\mathrm{S}_{\mathrm{a}}$ is the velocity vector defined as $\mathrm{Sa}=-\mathrm{e}^{\mathrm{Y}(\mathrm{r}) / 2} \delta^{0}{ }_{\mathrm{a}}$ Ricci scalar for the above metric Equation (2) is given by: 
$R=\frac{1}{2 r}\left[2 r Y^{\prime \prime}+r Y^{\prime 2}-r Y^{\prime} Z^{\prime}+2 Y^{\prime}-2 Z^{\prime}\right] e^{-Z}$

Using Equations (2-3) in Equation (1) after some calculations, we obtain following two Equations (5-6):

$\left[\frac{Y^{\prime} F}{2 r}-\frac{Y^{\prime} F^{\prime}}{2}+\frac{Z^{\prime} F}{2 r}+F^{\prime \prime}-\frac{Z^{\prime} F^{\prime}}{2}\right] e^{-Z}+k \rho=0$

$\left[\frac{Y^{\prime \prime} F}{2}+\frac{Y^{\prime 2} F^{\prime}}{4}-\frac{Y^{\prime} Z^{\prime} F}{4}-\frac{Y^{\prime} F^{\prime}}{2}+\frac{Z^{\prime} F}{2 r}+\frac{F^{\prime}}{r}\right] e^{-Z}+k \rho=0$

Our purpose is to solve Equations (5-6) which are highly non-linear, therefore it is very difficult to find the solution. Moreover, Equations (5-6) has three unknowns namely Y, $\mathrm{Z}$ and $\mathrm{F}$ Therefore, we need some extra condition to find the values of these unknowns. For this, we assume that $\mathrm{Y}^{\prime}=0$ so that Equations (5-6) after subtraction reduce into following Equation (7):

$\left[F^{\prime \prime}-\frac{Z^{\prime} F^{\prime}}{2}-\frac{F^{\prime}}{r}\right]=0$

To solve Equation (7) we use power law model of $f(R)$ i.e. $\mathrm{F}(\mathrm{R}) \propto \mathrm{f}_{0} \mathrm{R}^{\mathrm{m}}[24]$, where $\mathrm{f}_{0}$ and $\mathrm{m}$ are arbitrary constants. Hence,

$$
\left[R^{\prime \prime}+(m-1) \frac{R^{\prime 2}}{R}-\frac{Z^{\prime} R^{\prime}}{2}-\frac{R^{\prime}}{r}\right]=0
$$

with Ricci scalar $R=\frac{-Z^{\prime}}{r} e^{-Z}$. Assuminge $\mathrm{e}^{Z(\mathrm{r})}=\mathrm{r}^{-\alpha}$ where $\alpha$ is any real number, we have from Equation (8) that $\alpha(\alpha-$ 2) $\mathrm{r}^{\alpha-4}[3 \alpha-8+2(\alpha-2)(\mathrm{m}-1)]=0$ and the Ricci scalar $R$ takes the form $\mathrm{R}=\alpha \mathrm{r}^{\alpha-2}$ Here, following cases arise:

Case (a): $[3 \alpha-8=2(\alpha-2)(m-1)]=0$ but $\alpha(\alpha-2) r^{\alpha-4} \neq 0$.

Case (b): $\alpha(\alpha-2) r^{\alpha-4}=0$ but $[2 \alpha-8+(\alpha-2)(m-1)] \neq 0$.

We will discuss each case in detail.
Case (a): In this case, we have $[3 \alpha-8+2(\alpha-2)(\mathrm{m}-1)=0$ but $\alpha(\alpha-2) \mathrm{r}^{\alpha-4} \neq 0$, number of solutions could be obtained for different values of $\alpha$. For example, a solution is obtained for $\alpha=4$ which implies that $m=0$. In this case metric Equation (2) takes the form

$d S^{2}=\alpha_{1} d^{2}+r^{-4} d r^{2}+r^{2} d \theta^{2}$

and the value of Ricci scalar is $\mathrm{R}=4 \mathrm{r}^{2}$. Now, we are interested to find CKVFs of the above metric Equation (9). A vector field $X$ is said to be a conformal Killing vector field if [25].

$\mathrm{L}_{\mathrm{X}} \mathrm{g}_{\mathrm{ab}}=2 \eta \mathrm{g}_{\mathrm{ab}}$

Where $\eta=\eta(r), X, g_{a b}$ and $L$ represents conformal factor, vector field, metric tensor and Lie derivative operator respectively. Using Equation (10) in Equation (9), one obtains the following six first order non-linear PDEs (Partial Differential Equations):

$$
\begin{aligned}
& X_{, 0}^{0}=\eta \\
& -\alpha_{1} X_{, 1}^{0}+r^{-4} X_{, 0}^{1}=0 \\
& r^{2} X_{, 0}^{2}-\alpha_{1} X_{, 2}^{0}=0 \\
& -2 X^{1}+r X_{, 1}^{1}=\eta r \\
& X_{, 2}^{1}+r^{6} X_{, 1}^{2}=0 \\
& X^{1}+r X_{, 2}^{2}=\eta r
\end{aligned}
$$

Solving Equations (12-13) and Equation (15) by direct integration technique, we get the following information:

$$
\left.\begin{array}{l}
X^{0}=\frac{r}{\alpha_{1}} \int A^{1}(t, \theta) d \theta+\frac{1}{\alpha_{1}} \int r^{-4} A_{t}^{3}(t, r) d r+A^{2}(t, \theta) \\
X^{1}=r^{4} \iint A^{1}(t, \theta) d t d \theta-r^{6} \int A_{r}^{2}(r, \theta) d \theta+A^{3}(t, r) \\
X^{2}=\frac{1}{r} \int A^{1}(t, \theta) d t+A^{2}(r, \theta)
\end{array}\right\}
$$


Where $A^{1}(t, \theta), A^{2}(r, \theta)$ and $A^{3}((t, r)$ are arbitrary functions of integration to be determined. To find the values of these unknown functions of integration, we use the remaining Equations $(11,14)$ and Equation (16). Skipping lengthy but straightforward calculations, we obtain $\eta=c_{1}$ which means that conformal vector fields become homothetic vector fields which are:

$\left.\begin{array}{l}X^{0}=c_{1} t+c_{3} \\ X^{1}=-c_{1} r \\ X^{2}=2 c_{1} \theta+c_{8}\end{array}\right\}$

where $\mathrm{c}_{1}, \mathrm{c}_{3}, \mathrm{c}_{8} \in \mathfrak{R} /\{0\}$. Now, we find DKVFs of the metric Equation (9) which is defined as [13]

$\mathrm{L}_{\mathrm{K}} \mathrm{g}_{\mathrm{ab}}=2 \mathrm{mg}_{\mathrm{ab}}+2 \mathrm{pV}_{\mathrm{a}} \mathrm{V}_{\mathrm{b}}$

where ' $m$ ' and ' $p$ ' are scalar fields and $V$ is called null-like vector. Equivalently,

$\mathrm{L}_{\mathrm{K}} \mathrm{g}_{\mathrm{ab}}=2 \mathrm{mg}_{\mathrm{ab}}+2 \mathrm{pg}_{\mathrm{ac}} \mathrm{g}_{\mathrm{bd}} \mathrm{V}^{\mathrm{c}} \mathrm{V}^{\mathrm{d}}$

Assuming a null vector $V=\left(\sqrt{\frac{2}{\alpha_{1}}}, r^{2}, r^{-1}\right)$ for the metric Equation (9) and using Equation (20) one can obtain a system of six first order non-linear PDEs:

$K_{, 0}^{0}=m-2 p$

$\alpha_{1} r^{4} K_{, 1}^{0}-K_{, 0}^{1}=2 p r^{2} \sqrt{2 \alpha_{1}}$

$\alpha_{1} K_{, 2}^{0}-r^{2} K_{, 0}^{2}=2 p r \sqrt{2 \alpha_{1}}$

$-2 K^{1}+r K_{, 1}^{1}=2 r(m+p)$

$K_{, 2}^{1}+r^{6} K_{, 1}^{2}=2 p r^{3}$

$K^{1}+r K_{, 2}^{2}=r(m+p)$
Where $\mathrm{m}$ and $\mathrm{p}$ are functions of the radial coordinates ' $r$ '. Equations (21,24 and 26) gives the following information:

$$
\left.\begin{array}{l}
K^{0}=t(m-2 p)+B^{3}(r, \theta), K^{1}=r^{2} \int \frac{m+p}{r^{2}} d r+r^{2} B^{1}(t, \theta) \\
K^{2}=\theta(m-2 p)-r \theta \int \frac{m+p}{r^{2}} d r+\int B^{1}(t, \theta) d \theta+B^{2}(t, r)
\end{array}\right\}
$$

where $\mathrm{B}^{1}(\mathrm{t}, \theta), \mathrm{B}^{2}(\mathrm{t}, \mathrm{r})$ and $\mathrm{B}^{3}(\mathrm{r}, \theta)$ are functions of integration to be determined. Again, using some algebraic and direct integration techniques and avoiding tedious calculations, one finds that $\mathrm{p}=0$ and $\mathrm{m}=\mathrm{c}_{1}$ which implies that the DKVFs reduce into homothetic vector fields and are given in Equation (18).

Case (b): In this case, we have $\alpha(\alpha-2) r^{\alpha-4}=0$ but $[2 \alpha-8+$ $2(\alpha-2)(m-1)] \neq 0$. Now, if $\alpha(\alpha-2) r^{\alpha-4}=0$, then two subcases arise: (bi) $\alpha=0$ and (bii) $\alpha=2$ For the subcase (bi) i.e. $\alpha=0$ we have the following metric:

$\mathrm{ds}^{2}=\alpha_{1} \mathrm{dt}^{2}+\mathrm{dr}^{2}+\mathrm{r} 2 \mathrm{~d} \theta^{2}$

Using the procedure as given in case (a), one finds that $\eta=c_{2}$ which means that CKVFs become HVFs which are:

$$
\left.\begin{array}{l}
X^{0}=c_{2} t+\frac{r}{\alpha_{1}}\left(c_{7} \cos \theta+c_{8} \sin \theta\right)+c_{4} \\
X^{1}=c_{2} r+t\left(c_{7} \cos \theta+c_{8} \sin \theta\right)+c_{10} \cos \theta+c_{11} \sin \theta \\
X^{2}=\frac{1}{r}\left[t\left(-c_{7} \sin \theta+c_{8} \cos \theta\right)+\left(c_{10} \sin \theta+c_{11} \cos \theta\right)\right]+c_{9}
\end{array}\right\}
$$

wherec $_{2}, \mathrm{c}_{4}, \mathrm{c}_{7}, \mathrm{c}_{8}, \mathrm{c}_{9}, \mathrm{c}_{10}, \mathrm{c}_{11} \in \mathfrak{R} /\{0\}$. Next, we find DKVFs of the above metric Equation (28). For doing this, we define a null vector $v=\left(\sqrt{\frac{2}{\alpha_{1}}}, 1, r^{-1}\right)$ for the metric Equation (28) and using the definition of DKVF given in Equation (20), one finds that $\mathrm{p}=0$ and $\mathrm{m}=\mathrm{c}_{2}$ which means that the DKVFs reduce into homothetic vector fields and are given in Equation (29). For the subcase (bii) $\alpha=2$ we have the following metric:

$d S^{2}=-\alpha_{1} d t^{2}+r^{-2} d r^{2}+r^{2} d \theta^{2}$ 
Again using the definitions given in Equations (10 and 20) and skipping the details one finds that $p=m=\eta=0$ which means that both CKVFs and DKVFs become KVFs which are:

$$
\left.\begin{array}{l}
X^{0}=K^{0}=c_{4}, X^{1}=K^{1}=2 r \theta c_{1}-c_{2} r \\
X^{2}=K^{2}=-\left(\frac{r^{2} \theta^{2}}{r^{2}}\right) c_{1}+c_{2} \theta+c_{3}
\end{array}\right\}
$$

where $\mathrm{c}_{1}, \mathrm{c}_{2}, \mathrm{c}_{3}, \mathrm{c}_{4} \in \mathfrak{R} /\{0\}$.

\section{CONCLUSION}

In this paper, CKVFs and DKVFs structure of 3D circularly symmetric static metric in the $f(R)$ theory of gravity is presented. The purpose of this paper was twofold. Firstly, we have found some dust matter solutions of EFEs in the $f(R)$ theory of gravity and then we have extended our analysis by finding CKVFs and DKVFs of the resulting solutions. This study consists of two cases. Studying each case in detail, we find that in the case (a), both the CKVFs and DKVFs reduce into HVFs having dimension three see Equation(18). From these three HVFs two are KVFs and one is proper HVF. In the case (bi), both the CKVFs and DKVFs become HVFs having dimension seven; see Equation (29). Out of these seven HVFs six are KVFs one is proper HVF. In the case (bii), both the DKVFs and CKVFs become KVFs having dimension four; see Equation (31). Moreover, the use of null-like disformal transformation shows that the geometry of an object is changed and the background and foreground light cones coincides in the null direction. Actually, these are the preliminary results on DKVFs which may further be extended in four dimensions.

\section{ACKNOWLEDGEMENT}

The authors are highly thankful to Prof. Dr. Ghulam Shabbir, Faculty of Engineering Sciences, Ghulam Ishaque Khan Institute of Engineering Sciences \& Technology, Topi, Swabi, Peshawar, Pakistan, for useful discussions on DKVFs during this research work.

\section{REFERENCES}

[1] Sharif, M., and Majeed, B., "Teleparallel Killing Vectors of Spherically Symmetric Spacetimes", Communications in Theoretical Physics, Volume 52, No. 3, pp. 435-440, China, 2009.

[2] Hall, G.S., and Steele, J.D., "Conformal Vector Fields in General Relativity", Journal of Mathematical Physics, Volume 32, No. 7, pp. 1847-1853, USA, 1991.

[3] Maartens, R., Maharaj, S.D., and Tupper, B.O., "General Solution and Classification of Conformal Motions in Static Spherical Spacetimes", Classical and Quantum Gravity, Volume 12, No. 10, pp. 2577-2586, UK, 1995.

[4] Hall, G.S., and Capocci, M.S., "Classification and Conformal Symmetry in Three Dimensional space-times", Journal of Mathematical Physics, Volume 40, No. 3, pp. 1466-1478, USA, 1999.

[5] Berredo-Peixoto, G., "On the Static Solutions in Gravity with Massive Scalar Field in Three Dimensions", Classical and Quantum Gravity, Volume 20, No. 19, pp. 4305-4314, UK, 2003.

[6] Banados, M., Teitelboim, C., and Zanelli, J., "Black Hole in Three-Dimensional Spacetime", Physical Review Letters, Volume 69, No. 13, pp. 1849-1856, USA, 1992.

[7] Garcia, A.A., Hehl, F.W., Heinicke, C., and Macias, A., "Exact Vacuum Solution of (1+2)-Dimensional Poincaré Gauge Theory: BTZ Solution with Torsion", Physical Review-D, Volume 67, No. 12, Article ID 124016 pp. 1-7, USA, 2003.

[8] Sousa, A.A., and Maluf, J.W., "Black Holes in 2+1 Teleparallel Theories of Gravity", Progress of Theoretical Physics, Volume 108, No. 3, pp. 457-470, Japan, 2002.

[9] Bokhari, A.H., Karim, M., Al-Sheikh, D.N., and Zaman, F.D., "Circularly Symmetric Static Metric in Three Dimensions and its Killing Symmetry", International Journal ofTheoretical Physics, Volume 47, No. 10, pp. 2672-2678, USA, 2008

[10] Zhang, H., Liu, D.J., and Li, X.Z., "Black Holes and Gravitational Waves in Three Dimensional $\mathrm{f}(\mathrm{R})$ Gravity", Physical Review-D, Volume 90, No. 12, Article ID 124051, pp. 1-5, USA, 2014. 
[11] Ali, A., Rehman, S., Ramzan, M., and Khan, S., "Proper Homothetic Vector Fields on Three Dimensional Lorentzian Manifold of Circularly Symmetric Static Spacetimes", Sindh University Research Journal (Science Series), Volume 47, No. 4, pp. 739-742, Jamshoro, Pakistan, 2015.

Ramzan, M., Ahmed, Y., and Mufti, M. R.., "Ricci and Matter Collineations of Som-Roy Chaudhary Spacetime", Mehran University Research Journal of Engineering \& Technology, Volume 37, No. 2, pp. 315-318, Jamshoro, Pakistan, April, 2018.

[13] Lobo, I.P., and Carvalho, G.G., "The Geometry of NullLike Disformal Transformations", arXiv Preprint arXiv:1707.01784, 2017.

[14] Bekenstein, J.D., "Relativistic Gravitation Theory for Modified Newtonian Dynamics Paradigm”, Physical Review-D, Volume 70, No. 8, Article ID 083509, pp. 1-28, USA, 2004.

[15] Clifton, T., Ferreira, P.G., Padilla, A., and Skordis, C., "Modified Gravity and Cosmology", Physics Reports, Volume 513, No. 1-3, pp. 1-198, Netherlands, 2012.

[16] Koivisto, T.S., Mota, D.F., and Zumalacárregui, M., "Screening Modifications of Gravity through Disformally Coupled Fields", Physical Review Letters, Volume 109, No. 24, Article ID 241102, pp. 1-5, USA, 2012.

[17] Ip, H.Y., Sakstein, J., and Schmidt, F., "Solar System Constraints on Disformal Gravity Theories", Journal of Cosmology and Astroparticle Physics, No. 10, pp. 1-22, UK, 2015.
[18] Sakstein, J., "Disformal Theories of Gravity: From the Solar System to Cosmology", Journal of Cosmology and Astroparticle Physics, No. 12, pp. 1-29, UK, 2014.

[19] Sakstein, J., and Verner, S., "Disformal Gravity Theories: A Jordan Frame Analysis", Physical Review-D, Volume 92, No. 12, Article ID 123005, pp. 1-17, USA, 2015 .

[20] Kaloper, N., "Disformal Inflation", Physics Letters-B, Volume 583, No. 2, pp. 1-13, Netherlands, 2004.

[21] Carvalho, G.G., Lobo, I.P., and Bittencourt, E., "Extended Disformal Approach in the Scenario of Rainbow Gravity", Physical Review-D, Volume 93, No. 4, Article ID 044005, pp. 1-9, USA, 2016.

[22] Jumale, J.K., Teltumbade, D.P., and Thengane, K.D., "Six-Dimensional Static Plane Symmetric Vacuum Solutions in $\mathrm{f}(\mathrm{R})$ Gravity", Prespacetime Journal, Volume 3, No. 5, pp. 495-503, USA, 2012.

[23] Shamir, M.F., and Raza, Z., "Dust Static Cylindrically Symmetric Solutions in $\mathrm{f}(\mathrm{R})$ Gravity", Communications in Theoretical Physics, Volume 62, No. 3, pp. 348-352, China, 2014

[24] Nojiri, S.I., and Odintsov, S.D., "Unified Cosmic History in Modified Gravity: From $f(R)$ Theory to Lorentz NonInvariant Models", Physics Reports, Volume 505, No. 2, pp. 59-144, Netherlands, 2011.

[25] Hall, G.S., "Symmetries and Curvature Structure in General Relativity", World Scientific, Volume 46 , Singapore, 2004. 